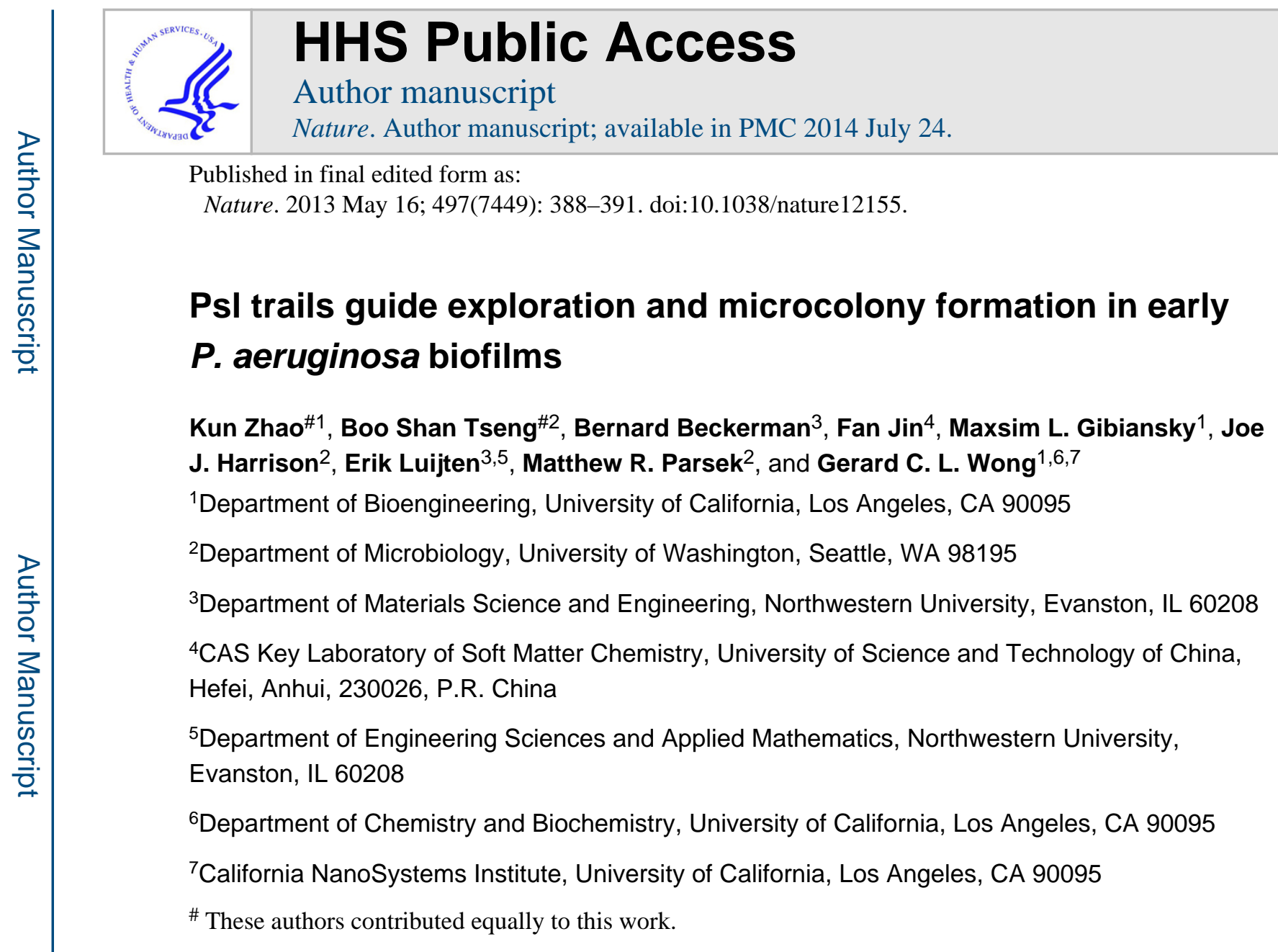

\title{
Abstract
}

Bacterial biofilms are surface-associated, multicellular, morphologically complex microbial communities ${ }^{1-7}$. Biofilm-forming bacteria such as the opportunistic pathogen ${ }^{7-10}$ Pseudomonas aeruginosa are phenotypically distinct from their free-swimming, planktonic counterparts. Much work has focused on factors impacting surface adhesion and it is known that $P$. aeruginosa secretes the Psl exopolysaccharide, which promotes surface attachment by acting as a 'molecular glue ${ }^{11-15}$. However, how individual surface-attached bacteria self-organize into microcolonies, the first step in communal biofilm organization, is not well understood. Here, we identify a new role for Psl in early biofilm development using a massively parallel cell-tracking algorithm to extract the motility history of every cell on a newly colonized surface via a search-engine based approach ${ }^{16}$. By combining these techniques with fluorescent Psl staining and computer simulations, we show that $P$. aeruginosa deposits a trail of Psl as it moves on a surface, which

\footnotetext{
Users may view, print, copy, download and text and data- mine the content in such documents, for the purposes of academic research, subject always to the full Conditions of use: http://www.nature.com/authors/editorial_policies/license.html\#terms

Correspondence and requests for materials should be addressed to G. C. L. Wong (gclwong@ seas.ucla.edu), M. R. Parsek (parsem@u.washington.edu) or E. Luijten (luijten@ northwestern.edu)..

Author contributions K.Z. and B.S.T. contributed equally to this work. G.C.L.W, M.R.P. and K.Z. conceived the project. K.Z., B.S.T., M.R.P., and G.C.L.W. designed studies. K.Z. and B.S.T. performed experimental measurements. K.Z. and G.C.L.W. performed image analysis. F.J. helped in performing image analysis. M.L.G. helped in collecting experimental data. B.S.T., J.J.H. and M.R.P. constructed strains. B.B. and E.L. designed the model and performed computer simulations. K.Z., B.S.T., B.B., E.L., M.R.P. and G.C.L.W. wrote the paper. All authors discussed the results and commented on the manuscript.

The authors declare no competing financial interests.

Supplementary Information is linked to the online version of the paper at www.nature.com/nature.
} 
influences the surface motility of subsequent cells that encounter these trails and thus generate positive feedback. Both experiments and simulations indicate that the web of secreted Psl controls the distribution of surface visit frequencies, which can be approximated by a power law. This Zipf's Law ${ }^{17}$ indicates that the bacterial community self-organizes in a manner analogous to a capitalist economic system ${ }^{18}$, a 'rich-get-richer' mechanism of Psl accumulation that results in a small number of 'elite' cells extremely enriched in communally produced Psl. Using engineered strains with inducible Psl production, we show that local Psl levels determine post-division cell fates and that high local Psl levels ultimately allow 'elite' cells to serve as the founding population for initial microcolony development.

The extracellular matrix of $P$. aeruginosa biofilms is composed mostly of exopolysaccharides (EPS), proteins and DNA ${ }^{19-21}$. Biofilms formed by non-mucoid isolates of $P$. aeruginosa primarily utilize two types of EPS: Psl and $\mathrm{Pel}^{22}$. To initiate biofilm development, the wild-type (WT) strain PAO1 uses Psl to promote adhesion of cells to surfaces $^{14,15}$. Once associated with a surface, $P$. aeruginosa can remain fixed at the point of attachment or move. Formation of microcolonies (aggregates of $\sim 50$ cells or less) is the first step in the communal organization of a biofilm, however little is known regarding the transition of individual cells to these discrete microcolonies ${ }^{3-5,23}$.

Consistent with the role of Psl in surface adhesion ${ }^{7,24}$, the average surface residence time of a PAO1 $\Delta p s l D$ mutant strain (which cannot produce Psl) was $35 \pm 10 \%$ shorter than that of WT. Tracking algorithms allow us to isolate differences in spatial characteristics of cellsurface interactions, in addition to temporal characteristics such as residence time. We observed a fundamental difference in surface exploration patterns between these strains (Figure 1a-d, Supplementary Video 1) when we tracked the motility history of all cells in a $67 \times 67 \mu \mathrm{m}^{2}$ field of view (>700,000 images of individual cells, 11 hours of data, 3 second/ frame time resolution). In Figures 1a-d, black regions represent 'untouched' surface areas, while red regions represent areas visited by bacteria. For WT, the surface coverage increased slowly to a maximum of $\sim 55 \pm 5 \%$ in 5 hours. In contrast, the $\Delta p s l D$ mutant covered $79 \pm$ $10 \%$ in 5 hours. These observed differences are not due to differences in growth between strains (Supplementary Figure 1). To confirm that changes in surface motility rather than changes in the numbers of bacteria visiting the surface are responsible for these observations, we also compared WT and $\triangle p s l D$ at the same total number of bacterial visits (i.e. the sum of the number of bacteria in all frames $N_{\mathrm{S}}=\Sigma n_{i}$, where $n_{i}$ is the number of bacteria in frame $i$; Supplementary Figure 2). Indeed, essentially the same trend was observed, with a surface coverage of $52 \pm 4 \%$ for WT at $N_{\mathrm{s}}=124,000$ and a surface coverage of $83 \pm 10 \%$ for $\Delta p s l D$ at the same $N_{\mathrm{s}}$. These are averages from at least three replicates (see Supplementary Methods).

Since Psl plays an important role in both surface motility and adhesion, we characterized its spatial distribution with respect to bacterial trajectories. A TRITC-conjugated, Psl-specific lectin ${ }^{12}$ was used to visualize Psl. We allowed WT cells to traverse a surface for 2 hours before lectin staining and found that a trail of Psl was left on the surface (Supplementary Figure 3). We verified that eDNA ${ }^{21}$ was not found in the trails (by propidium iodide (data not shown) and Syto9 staining (Supplementary Figures 4 and 5)). 
Cell-tracking algorithms and lectin staining were combined to investigate the spatiotemporal aspects of Psl deposition in a manner that accounts for the changing speeds (and thereby residence times) of cells. Figure 1e shows bacterial trajectories between times $t_{1}$ (16.3 hours after inoculation) and $t_{2}$ (18.7 hours after inoculation). Figure 1f shows the lectin-stained Psl trails generated in this time period (see Methods, Supplementary Figure 6). Surface regions with the brightest fluorescence signals, and hence the highest local surface concentrations of Psl, corresponded to those with the longest bacterial visits. Multiple visits to a surface region by different cells and extended residence times of a specific cell on the same surface region both served to increase local Psl concentration.

We used cell-tracking algorithms to determine the bacterial visit frequency for each surface pixel for the entire community (see Methods). A surface visit map (Figure 2a) of all WT cells within the field of view (Figure $2 b, \sim 15.7$ hours after inoculation) shows that a large fraction of the surface was never visited. Of the surface areas traversed by bacteria, most were traversed once. In fact, the visit frequency distribution (histogram of the number of pixels with $N$ bacterial visits) was measured to be a monotonically decreasing function of $N$. The precise form of the function is complex; however, for $N$ ranging from a few visits to over a hundred visits, the distribution is approximately described by a power law with exponent of $-2.9 \pm 0.1$ (Figure 2c), which serves as a simple metric for the distribution.

The importance of Psl in determining this global pattern of bacterial surface exploration can be seen in the visit histograms of the $\Delta \mathrm{P}_{p s} / \mathrm{P}_{B A D^{-}} p s l$ mutant strain, which employs an arabinose-inducible promoter to control Psl production ${ }^{25}$. As the concentration of arabinose in the growth medium was changed from $0 \%$ to $1 \%(\mathrm{w} / \mathrm{v})$, the visit histograms showed a systematic shift from a more uniform distribution of surface visits (i.e. steep power-law decay) to a more hierarchical distribution (i.e. having a broader range of visit frequencies) (Figure $2 \mathrm{~d}$ ), as evidenced by the change in the effective power-law exponent from $-3.3 \pm 0.1$ to $-1.9 \pm 0.1$, which correspond to low and high arabinose-induced Psl production, respectively. Since the total number of visits is the same for the different curves, a less steep power-law behavior implies that with increasing Psl concentration the bacterial visits become concentrated in a smaller fraction of the surface. These observations lead us to hypothesize that the higher the Psl concentration for a given surface region, the more likely this region will be visited by more bacteria, causing further local accumulation of Psl and resulting in positive feedback.

Type IV pili (TFP) play a pivotal role in $P$. aeruginosa surface motility and the power-law exploratory mechanism. TFP can extend considerably from the cell body ${ }^{26}$ and assist in "exploring" surface areas beyond the cellular 'footprint'. We hypothesize that TFP associate with Psl-rich surface regions and pull the cell toward these regions with higher probability. Consistent with this, we show that a $\triangle$ pilA mutant strain, which eliminates TFP-driven twitching motility, results in a drastic reduction of surface exploration (Supplementary Figure 7). Since cells can secrete Psl as well as associate with Psl, twitching motility effectively promotes positive feedback. Simple track following is but one possible manifestation of this mechanism: Cells can approach a given track from any direction. While such cells can move toward Psl, they do not necessarily re-orient themselves tangentially to a given track. 
Power law relationships generally exist only over a limited range in nature and are often difficult to distinguish from other quantitatively similar relationships. Therefore, rather than concentrating on the approximate power law in the visit frequency distribution and the range over which it is observed, we focused instead on obtaining a deeper quantitative understanding of the processes that generate its functional form. Thus, we performed computer simulations of Psl-guided motility of Psl-secreting bacteria using experimentally measured parameters to stringently test the quantitative interplay between progressive Psl secretion, surface motility and the idea of surface exploration guided by positive feedback. Bacteria were modeled as non-overlapping line segments in a two-dimensional domain that, when unbiased by Psl, move according to a velocity distribution extracted from the experimental data for $\Delta \mathrm{P}_{p s l} / \mathrm{P}_{B A D}-p s l$ at $0 \%$ arabinose. In each step, a fixed amount of Psl was secreted and the bacterial displacement was biased by the local Psl distribution. The bacterial concentration and dimensions as well as the sampling rate were all chosen in accordance with the experimental parameters (see Supplementary Methods). The simulations captured the complex distributions from experiments, in both the power-law regime and beyond (Figures 2e and 3c), including the dependence on Psl. The simulated visit frequency distributions exhibited a power-law behavior that agrees quantitatively with the tracked microscopy data. This is a striking confirmation of the role of Psl-biased motion as the underlying mechanism, since unbiased motion would give rise to nonlocalized, random-walk type behavior. As the Psl production rate was increased for both experiments (Figure 2d) and simulations (Figure 2e), the exponents increased over the same numerical range, from -3.1 to -1.8 , confirming the shift to more hierarchical distributions in which the number of highly visited sites increased at the expense of visits to many of the rarely visited sites (Figures 2f-h). Likewise, both the lectin-stained Psl image observed in experiments (Figure 2i) and the simulated Psl map (Figure 2j) showed the hierarchical nature of the spatial distribution of Psl.

Power laws (Zipf's Law ${ }^{17,18}$ or Pareto distributions, its continuous generalization) are known to describe self-organized systems like the wealth distributions in capitalist economies ${ }^{18}$. We found that bacteria are guided by synergistic 'rich-get-richer' mechanisms. Both simulations and experiments indicate that $P$. aeruginosa's ability to secrete Psl and its tendency to associate with Psl result in an exploratory strategy in which cells go where other cells go most often. (Interestingly, the range of exponents we observe here for bacteria is essentially the same as for income distributions ${ }^{27}$.) This strategy results in a small number of cells positioned at locations that are extremely enriched in communally produced Psl. This arrangement provides the conditions necessary for microcolony growth. Importantly, these effects persist even when we completely turn off cell division in computer simulations, which indicates that the distribution of cells is a consequence of their exploratory strategy rather than of growth.

We examined the behavior of bacterial communities beyond the power-law exploratory phase to show how growth can amplify differences in cell density on the surface caused by exploration. We found that high local Psl levels resulted in discrete microcolonies that exhibit localized exponential growth rather than delocalized surface exploration. For WT data (Figures 2a-c), the power-law behavior persisted only in the initial exploratory phase 
when there were no microcolonies. In fact, small deviations from the power law can be observed at the lower right portion of Figure 2c (green arrow), which corresponds to highvisit areas with the highest local Psl concentrations. To examine community behavior near high Psl concentrations, we examined the $\Delta \mathrm{P}_{p s} / \mathrm{P}_{B A D^{-}}$psl mutant that overproduces Psl in the presence of $1 \%$ arabinose (Psl++). The bacterial visit frequency distribution for the Psl+ + cells was significantly different from that of WT cells (Figure 3a). Compared to Figure 2a, there are far fewer tracks leading into and out of the cellular aggregates in Figure 3a, so few cells joined or departed from these aggregates. The regions of highest bacterial visits in the map fully correlate with the existence of microcolonies of closely packed bacteria in the bright field image (Figure 3b). The cell densities in the high-visit areas were more than three times higher than the average cell density on the surface. Moreover, the Psl++ cells formed microcolonies significantly earlier than the WT. Importantly, due to microcolony formation, a significant portion of the visit frequency distribution is described by an exponential rather than a power law in both experiments and simulations (Figure 3c).

We used cell tracking to show that the exponential growth that enables localized microcolony formation is ultimately rooted in how local Psl concentrations determine postdivision cell fates. We distinguished three possibilities: the post-division cells either 1) both 'stay' near the division event or 2) both 'leave' (see definitions of 'stay' and 'leave' in Supplementary Methods) or 3) one cell 'stays' and the other 'leaves'. Figure 3d shows the probabilities of these cell fates observed for WT, Psl++ and $\Delta p s l D$. The probability for both cells staying for the Psl++ mutant was approximately three times as high as for WT. In contrast, the probability for both cells staying for the $\triangle p s l D$ mutant strain was essentially zero. In fact, the fraction of total population growth due to cell division (as opposed to motile cells joining the microcolony, cells attaching from solution, etc.) was $\sim 85 \%$ for the Psl++ mutant, which directly leads to an exponential visit distribution (see Supplementary Figure 8). We traced the division lineage of cells in developing microcolonies (Figure 3e). Clearly, the Psl++ microcolony was derived mostly from a single lineage, consistent with our proposal that such cells primarily divide and remain in the same area due to the high local Psl concentrations. In contrast, the WT microcolony developed from many cell lineages due to the initial power-law exploratory phase. (More examples can be found in Supplementary Figures 9 and 10). These data strongly support our 'rich-get-richer' model for microcolony formation.

The results presented here clearly and quantitatively show the key steps important for the transition from surface attachment and surface exploration to microcolony formation: the evolving distribution of Psl on a solid surface provides a mechanism for bacteria to selforganize socially, resulting in a 'rich-get-richer' power-law distribution of visit frequencies and a hierarchical distribution of Psl concentrations on the surface. This in turn results in a small number of aggregated cells highly enriched in Psl, which enables them to serve as the founding population for localized exponential growth and formation of microcolonies that ultimately evolve into the mushroom-like 'stalks'-and-'caps' morphology of mature biofilms $^{4-6}$. 


\section{Methods (online)}

\section{Strains and growth conditions}

P. aeruginosa strain PAO1 wild-type (WT) ${ }^{28}$ and its isogenic mutants $\Delta p s l D$, the pslinducible strain $\Delta \mathrm{P}_{p s} / \mathrm{P}_{B A D}$-psl and $\Delta p i l A$ were used in this study. The growth rates of the strains were nearly identical (to within measurement error, Supplementary Figure 1). For strain $\Delta \mathrm{P}_{p s} / \mathrm{P}_{B A D^{-}}$sl , different amounts of arabinose (Sigma-Aldrich; $1 \%, 0.1 \%$ and $0.005 \%$ were used in this work) were added into the medium to control the production of $\mathrm{Ps}^{14}$.

FAB medium ${ }^{29}$ with $0.6 \mathrm{mM}$ glutamate (Sigma-Aldrich) as the carbon source was used for flow cell experiments. An inoculum was prepared by growing strains in test tubes containing FAB with $30 \mathrm{mM}$ glutamate, shaking at $220 \mathrm{rpm}$ and $37^{\circ} \mathrm{C}$ to $\mathrm{OD}_{600} \approx 0.5$. Cultures were then diluted to $\mathrm{OD}_{600} \approx 0.01$ in $\mathrm{FAB}$ medium with $0.6 \mathrm{mM}$ glutamate, which was used for injection into the flow chamber.

\section{Flow cell assembly, sterilization and washing of the system}

The flow cell was purchased from the Department of Systems Biology, Technical University of Denmark and assembled as previously described ${ }^{30}$. The assembled flow cell was connected to a syringe through a $0.22 \mu \mathrm{m}$ filter (Fisher Scientific) using silicon tubing (Dow Corning). Then the whole system was sterilized overnight with $3 \% \mathrm{H}_{2} \mathrm{O}_{2}$ (Fisher Scientific) at $3 \mathrm{ml} /$ hour using a syringe pump (KD Scientific). After the sterilization, autoclaved DI water was used to wash the whole system overnight. The system was washed again using FAB medium with $0.6 \mathrm{mM}$ glutamate before starting the inoculation of the bacteria into the flow cell. The flow was stopped for bacterial inoculation and 20 minutes of incubation, then resumed with the initiation of image recording. Different flow rates have recently been reported to have an effect on bacterial surface behavior ${ }^{31}$, and it will be interesting to using large scale tracking methods in this context. In this work, the flow cell experiments were conducted at $30^{\circ} \mathrm{C}$ with a flow rate of $3 \mathrm{ml} /$ hour.

\section{Data acquisition}

The images were taken by an EMCCD camera (Andor iXon) using IQ software (Andor) on an Olympus IX81 microscope equipped with Zero Drift autofocus system. The bright-field images were recorded every 3 seconds for a total recording time of about 20 hours (varied by strain). Each data set, which had 18,000-48,000 frames, contained up to 1,000,000 bacteria images. The image size is $67 \times 67 \mu \mathrm{m}^{2}\left(1024 \times 1024\right.$ pixel $\left.^{2}\right)$.

\section{Lectin staining}

The Psl trails left by bacteria were stained with TRITC-labeled Hippeastrum hybrid lectin (HHA) from amaryllis (EY laboratories) ${ }^{12}$. Flow was suspended and $0.3 \mathrm{ml}$ of $100 \mu \mathrm{g} / \mathrm{ml}$ TRITC-HHA in FAB medium with $0.6 \mathrm{mM}$ glutamate was injected upstream of the inlet flow. During the injection, the flow chamber was monitored through the camera to make sure there was no shift or disturbance of the stage due to injection. The flow chamber was stained for 15 minutes without flow and then washed for 15 minutes with flow at a flow rate of $3 \mathrm{ml} /$ hour in the dark before imaging. 


\section{Image analysis, surface coverage map and visit frequency map}

Images were analyzed as previously described ${ }^{16}$. In Figures 1a-e we used the area of each moving cell to generate the surface coverage from the bacterial trajectories. The visit frequency distributions are generated at full data resolution; for the visit maps, we use the center of the cell to mark the trail, and smeared each of these marks to a square patch 8 pixels $(\sim 0.5 \mu \mathrm{m})$ wide, in accordance with bacterium width. The distribution of Psl on the PAO1 surface is expected to be more complex ${ }^{32}$, but this will amount to a more complex point spread function and is not expected to alter the results significantly.

\section{Simulation of Psl-guided motility of Psl-secreting bacteria}

The computer simulations employed a two-dimensional model in which an exponentially growing number of line segments of unit length moved within a square, periodic domain of side length 35, approximately corresponding to the experimental viewing window. Each bacterium was assigned a fixed step size and stepped with a variable angle. Both the step size and stepping angle were drawn from the experimentally determined speed distribution for the $\Delta \mathrm{P}_{p s l} / \mathrm{P}_{B A D^{-}} p s l$ strain at an arabinose concentration of $0 \%$ (i.e. in the absence of Psl). Starting from a random non-overlapping configuration, all bacteria propagated in discrete time steps $(\Delta t=3 \mathrm{~s})$ either moving at their constant, assigned rate, keeping their director fixed, or randomly reorienting. Reorientations occurred at a frequency $3.7 \%$ determined by the mean-square displacement of the bacteria in experiment. The effect of arabinose was represented by letting each bacterium deposit Psl at its center at a tunable rate. The surface accumulation of Psl was recorded in elementary squares of side length 1/29 (corresponding to experimental pixel size). The motion was in turn biased according to the presence of Psl within the vicinity of the leading pole of the bacterium. The total simulation time was chosen such that the final visit count was the same as in experiments. Over this window, we sampled individual bacterial trajectories as well as visit frequencies of all pixels (details in Supplementary Methods).

\section{Supplementary Material}

Refer to Web version on PubMed Central for supplementary material.

\section{Acknowledgements}

K.Z., B.S.T., M.R.P., and G.C.L.W. are supported by National Institutes of Health (NIH 1RO1HL087920). K. Z. and G.C.L.W. also acknowledge support from National Science Foundation (NSF DMR1106106) and an UCLA Transdisciplinary Seed Grant. B.S.T., J.J.H. and M.R.P. also acknowledge support from the NIH (R01 AI077628, R01AI081983, R56AI061396) and NSF (MCB0822405). B.S.T. is supported by the Cystic Fibrosis Foundation Postdoctoral Fellowship (TSENG11F0). J.J.H. was supported by a postdoctoral fellowship from the Natural Sciences and Engineering Research Council (NSERC) of Canada. B.B. and E.L. acknowledge support from the National Science Foundation under DMR-1006430 (E.L.) and DGE-0824162 (B.B.). The authors would like to thank Dr. Jernej Copic from the UCLA Economics Department for discussions and R.J. Siehnel for technical assistance.

\section{References}

1. Costerton JW, Stewart PS, Greenberg EP. Bacterial biofilms: a common cause of persistent infections. Science. 1999; 284:1318-22. [PubMed: 10334980] 
2. O'Toole GA, Kolter R. Flagellar and twitching motility are necessary for Pseudomonas aeruginosa biofilm development. Mol Microbiol. 1998; 30:295-304. [PubMed: 9791175]

3. O'Toole G, Kaplan HB, Kolter R. Biofilm formation as microbial development. Annu Rev Microbiol. 2000; 54:49-79. [PubMed: 11018124]

4. Stoodley P, Sauer K, Davies DG, Costerton JW. Biofilms as complex differentiated communities. Annu Rev Microbiol. 2002; 56:187-209. [PubMed: 12142477]

5. Klausen M, Aaes-Jørgensen A, Molin S, Tolker-Nielsen T. Involvement of bacterial migration in the development of complex multicellular structures in Pseudomonas aeruginosa biofilms. Mol Microbiol. 2003; 50:61-8. [PubMed: 14507363]

6. Davies DG, et al. The involvement of cell-to-cell signals in the development of a bacterial biofilm. Science. 1998; 280:295-8. [PubMed: 9535661]

7. Mann EE, Wozniak DJ. Pseudomonas biofilm matrix composition and niche biology. FEMS Microbiol Rev. 2012; 36:893-916. [PubMed: 22212072]

8. Lyczak JB, Cannon CL, Pier GB. Establishment of Pseudomonas aeruginosa infection: lessons from a versatile opportunist. Microbes Infect. 2000; 2:1051-60. [PubMed: 10967285]

9. Tatterson LE, Poschet JF, Firoved A, Skidmore J, Deretic V. CFTR and pseudomonas infections in cystic fibrosis. Front Biosci. 2001; 6:D890-7. [PubMed: 11487480]

10. Singh PK, et al. Quorum-sensing signals indicate that cystic fibrosis lungs are infected with bacterial biofilms. Nature. 2000; 407:762-4. [PubMed: 11048725]

11. Friedman L, Kolter R. Two genetic loci produce distinct carbohydrate-rich structural components of the Pseudomonas aeruginosa biofilm matrix. J Bacteriol. 2004; 186:4457-65. [PubMed: 15231777]

12. Ma LY, Lu HP, Sprinkle A, Parsek MR, Wozniak DJ. Pseudomonas aeruginosa PSl is a galactoseand mannose-rich exopolysaccharide. J Bacteriol. 2007; 189:8353-6. [PubMed: 17631634]

13. Byrd MS, et al. Genetic and biochemical analyses of the Pseudomonas aeruginosa Psl exopolysaccharide reveal overlapping roles for polysaccharide synthesis enzymes in Psl and LPS production. Mol Microbiol. 2009; 73:622-38. [PubMed: 19659934]

14. Ma L, Jackson KD, Landry RM, Parsek MR, Wozniak DJ. Analysis of Pseudomonas aeruginosa conditional psl variants reveals roles for the psl polysaccharide in adhesion and maintaining biofilm structure postattachment. J Bacteriol. 2006; 188:8213-21. [PubMed: 16980452]

15. Byrd MS, Pang B, Mishra M, Swords WE, Wozniak DJ. The Pseudomonas aeruginosa exopolysaccharide Psl facilitates surface adherence and NF-kappaB activation in A549 cells. mBio. 2010; 1:e00140-10. [PubMed: 20802825]

16. Gibiansky ML, et al. Bacteria use type IV pili to walk upright and detach from surfaces. Science. 2010; 330:197. [PubMed: 20929769]

17. Newman MEJ. Power laws, Pareto distributions and Zipf's law. Contemp Phys. 2005; 46:323-51.

18. Gabaix X. Power laws in economics and finance. Annu Rev Econ. 2009; 1:255-93.

19. Eagon RG. Composition of an extracellular slime produced by Pseudomonas aeruginosa. Can J Microbiol. 1962; 8:585-6.

20. Sutherland IW. The biofilm matrix--an immobilized but dynamic microbial environment. Trends Microbiol. 2001; 9:222-7. [PubMed: 11336839]

21. Whitchurch CB, Tolker-Nielsen T, Ragas PC, Mattick JS. Extracellular DNA required for bacterial biofilm formation. Science. 2002; 295:1487. [PubMed: 11859186]

22. Colvin KM, et al. The Pel and Psl polysaccharides provide Pseudomonas aeruginosa structural redundancy within the biofilm matrix. Environ Microbiol. 2012; 14:1913-28. [PubMed: 22176658]

23. Monroe D. Looking for chinks in the armor of bacterial biofilms. PLoS Biol. 2007; 5:e307. [PubMed: 18001153]

24. Flemming HC, Neu TR, Wozniak DJ. The EPS matrix: the "house of biofilm cells". J Bacteriol. 2007; 189:7945-7. [PubMed: 17675377]

25. Newman JR, Fuqua C. Broad-host-range expression vectors that carry the L-arabinose-inducible Escherichia coli araBAD promoter and the araC regulator. Gene. 1999; 227:197-203. [PubMed: 10023058] 
26. Skerker JM, Berg HC. Direct observation of extension and retraction of type IV pili. Proc Natl Acad Sci U S A. 2001; 98:6901-4. [PubMed: 11381130]

27. Solomon S, Richmond P. Power laws of wealth, market order volumes and market returns. Phys. A. 2001; 299:188-97.

28. Holloway BW. Genetic recombination in Pseudomonas aeruginosa. J Gen Microbiol. 1955; 13:572-81. [PubMed: 13278508]

29. Heydorn A, et al. Experimental reproducibility in flow-chamber biofilms. Microbiology. 2000; 146( Pt 10):2409-15. [PubMed: 11021917]

30. Sternberg C, Tolker-Nielsen T. Growing and analyzing biofilms in flow cells. Curr Protoc Microbiol. 2006 Chapter 1, Unit 1B 2.

31. Lecuyer S, et al. Shear stress increases the residence time of adhesion of Pseudomonas aeruginosa. Biophys J. 2011; 100:341-50. [PubMed: 21244830]

32. Ma L, et al. Assembly and development of the Pseudomonas aeruginosa biofilm matrix. PLoS Pathog. 2009; 5:e1000354. [PubMed: 19325879] 

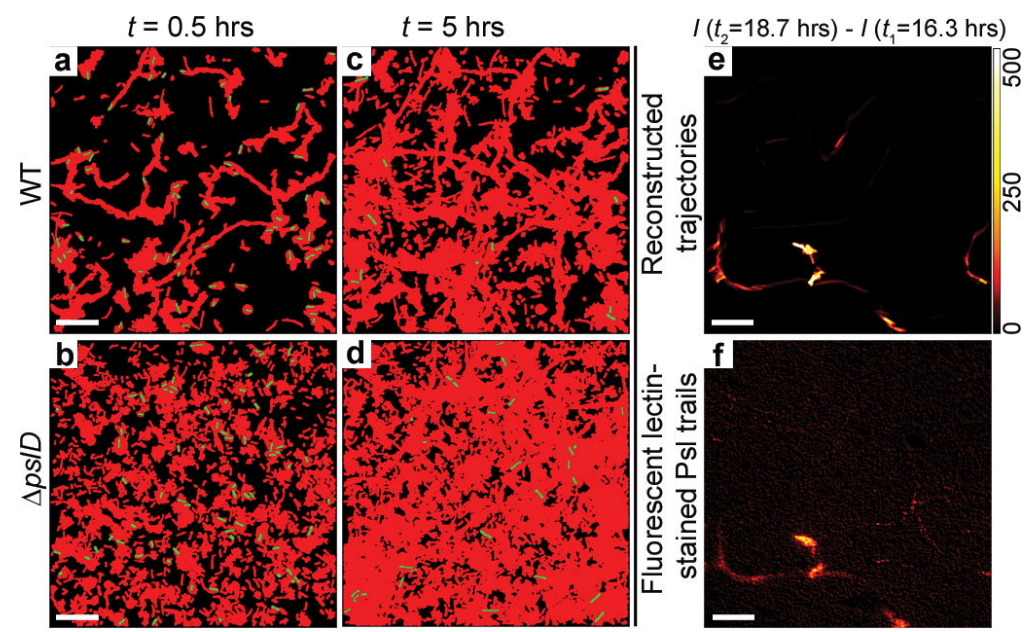

Figure 1. Efficiency of surface coverage by bacterial trajectories and correlation with Psl trails a-d. Cumulative surface coverage at $0.5(\mathbf{a}, \mathbf{b})$ and 5 hours $(\mathbf{c}, \mathbf{d})$. Top row is for WT and bottom row for $\Delta p s l D$. Red and black colors indicate used (i.e. covered by bacterial trajectories) and fresh surface, respectively. Bacteria in the current frame are shown in green. e. Reconstructed bacterial trajectories of WT generated between 16.3 and 18.7 hours after inoculation (color bar indicates the time a given cell spent at each point). f. Psl trail left behind by bacteria in the same period, stained by fluorescently conjugated HHA lectin. Scale bars are $10 \mu \mathrm{m}$. 

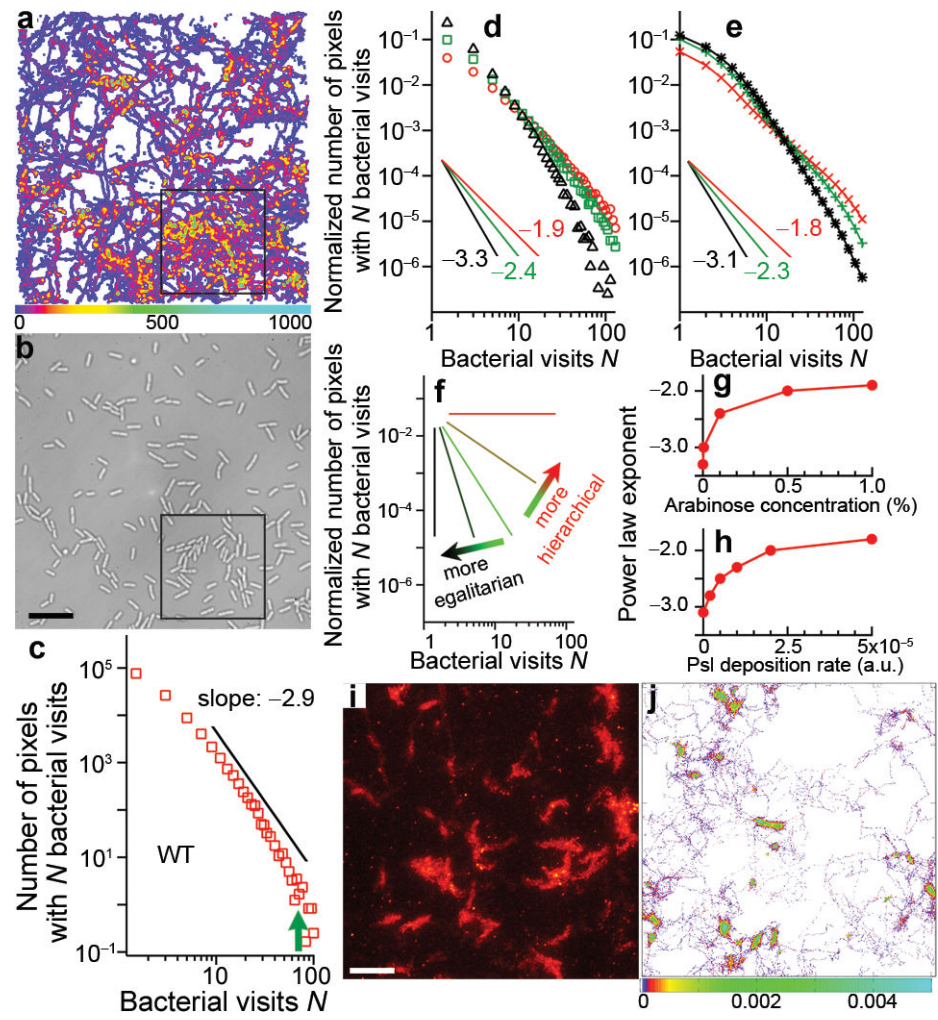

Figure 2. Visit frequency distribution and its effect on bacterial movement

a. Visit frequency map of WT for the first 15.7 hours post inoculation, when microcolonies were just starting to form (example outlined by black square). b. Bright-field image for WT at $t \sim 15.7$ hours. c. Visit frequency distribution from (a). Solid line shows a power-law decay with exponent -2.9 . Green arrow indicates where the curve begins to deviate from this power law. d. Visit frequency distributions for $\Delta \mathrm{P}_{p s} / \mathrm{P}_{B A D^{-}}$psl at arabinose concentrations $0 \%(\Delta), 0.1 \%(\square), 1 \%(\bigcirc)$. e. Simulation results of visit frequency distributions at Psl deposition rates (arbitrary units, see Supplementary Methods) $0\left(^{*}\right), 10^{-5}(+), 5 \times 10^{-5}(\times)$. In (d) and (e), each data set is normalized by the total number of visits (roughly the same as for (a)) and solid lines show power-law decay. f. Schematic graph showing that distributions with steep slopes are more egalitarian, while those with shallow slopes are more hierarchical. g./h. Fitted power-law exponents of visit frequency distributions from experiments at different arabinose concentrations (g) and simulations at different Psl deposition rates $(\mathbf{h})$. i. Fluorescent lectin-stained image showing hierarchical distribution of Psl $\left(\Delta \mathrm{P}_{p s l} / \mathrm{P}_{B A D^{-}} p s l\right.$ at $1 \%$ arabinose). j. Psl map from simulations (Psl deposition rate $5 \times$ $\left.10^{-5}\right)$. Scale bars are $10 \mu \mathrm{m}$. 


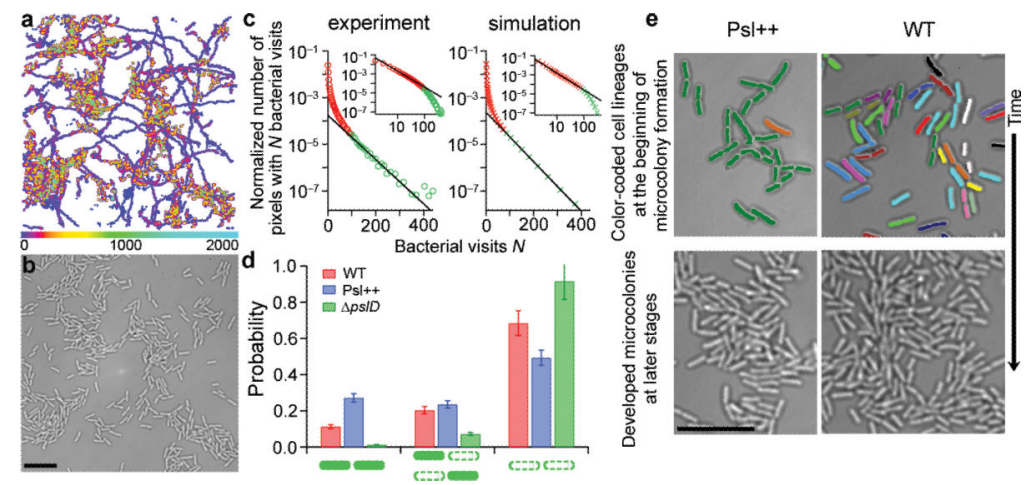

Figure 3. Local Psl levels determine post-division cell fates

a. Visit frequency map of Psl++ for the first 14 hours post inoculation. Microcolonies have already started to form. b. Bright-field image for Psl++ at $t \sim 14$ hours. c. Visit frequency distributions of Psl++ from (a) for experiments (left) and simulations (right) agree. Solid line is an exponential fit to the second part of the data (green). Inset shows a power-law fit to the first part of the data (red). d. Probability of post-division cells' fates: "stay" (solid rod) or "leave" (dashed rod envelope) for WT (red), Psl++ (blue) and $\Delta p s l D$ (green). Error bars are estimated from $1 / \widehat{ } N_{\text {div }}$, with $N_{\text {div }}$ the total number of division events during the time period of interest $\left(N_{\mathrm{div}} \geq 90\right)$. e. WT and Psl++ microcolonies have drastically different compositions, as depicted by color-coded cell division lineages at early stages of microcolony formation (top row). For Psl++ the microcolony is dominated by a single lineage, whereas the WT microcolony has 20 different lineages. Bottom row depicts more developed microcolonies at the same location 3.3 hours later. Scale bar is $10 \mu \mathrm{m}$. 